\title{
Smart Recognition COVID-19 System to Predict Suspicious Persons Based on Face Features
}

\author{
Mossaad Ben Ayed ${ }^{1,2} \cdot$ Ayman Massaoudi $^{3,4}$. Shaya A. Alshaya ${ }^{5}$
}

Received: 15 September 2020 / Revised: 23 November 2020 / Accepted: 22 January 2021 / Published online: 15 February 2021

(c) The Korean Institute of Electrical Engineers 2021

\begin{abstract}
The coronavirus (COVID-19) is identified at first in Wuhan in December 2019. The apparition of the COVID-19 virus is widely spread to concern all countries worldwide. The World Health Organization (WHO) on March 11 declare COVID-19 a pandemic. This Virus causes a serious infection of the respiratory system. Its high transmission constitutes great problems and challenges. The WHO proposes many actions to limit the spread of the virus such as quarantine and decrease or halt flights between states. The actions taken by states in airports are to detect suspicious persons with COVID-19. We aimed to provide a Computer-Aided Diagnosis (CAD) framework to predict suspicious COVID-19 person. This prediction identifies suspicious persons who suffer from shortness breath which is the main symptom of this disease. Extract shortness breath anomaly through the estimated heart rate from face based-video is the main contribution of the present paper. We developed a Smart Recognition COVID-19 (SRC) system to estimate the breath score. In conclusion, our study achieves an accurate breath score. The error is about 1 breath per minute. The proposed solution is of great importance because it helps managers in the airport to predict suspicious COVID-19 passengers.
\end{abstract}

Keywords COVID-19 $\cdot$ SARS-CoV-2 $\cdot$ Suspicious passenger $\cdot$ Video processing $\cdot$ Hear rate estimation $\cdot$ Breath score estimation

Mossaad Ben Ayed

mm.ayed@mu.edu.sa; mossaad_benayed@yahoo.fr

Ayman Massaoudi

ahmassaaoudi@ju.edu.sa; aymen.massaoudi@supcom.tn

Shaya A. Alshaya

shaya@mu.edu.sa

1 Computer Science Department, College of Sciences and Humanities Sciences At alGhat, Majmaah University, Majmaah 11952, Saudi Arabia

2 Computer and Embedded System Laboratory, Sfax University, Sfax Sfax, Tunisia

3 Department of Computer Science, Jouf University, Al Jouf, Sakaka 74331, Saudi Arabia

4 Department of Computer Science, Mediatron Lab, Sup'Com, Carthage University, 1054 Tunis, Tunisia

5 Computer Science Department, College of Sciences and Humanities Sciences At alGhat, Majmaah University, Majmaah 11952, Saudi Arabia

\section{Introduction}

The coronavirus 2 (SARS-CoV-2) called COVID-19 appeared in Wuhan China since December 2019. This new virus damages severely the respiratory system and led to a shortness breath [1]. The COVID-19 is quickly spread through other countries. At the end of September 2020, there are more than 30 million coronavirus cases in which more than 900000 deaths. An average of one hundred thousand cases per day and an average of three thousand deaths per day. These statistics prove the seriousness of the situation. It is affecting 215 countries and territories around the world [2]. The main problem of this virus compared with previous viruses is the increased spread rate [3-5].

Many states attempt to reduce the spread rate by applying confinement and quarantine strategies. Airports and hospitals perform preventive systems to identify COVID-19 persons and separate them from health persons $[4,6]$. Despite all these preventions, many countries that have advanced health-care systems as China, Italy, USA, Spain, and France are unable to support the increasing cases of COVID-19 [2, $6]$. 
The COVID-19 is characterized by three essential symptoms: fever, cough, and shortness of breath [7]. This paper tries to identify an infected passenger with COVID-19 based on the Breath Score (BS) as an indication of the shortness of breath. Most airports used a thermal camera to read the passenger's temperature to identify suspicious ones. Unfortunately, some passengers who did not want to be confined take paracetamol to avoid the fever. Coughs have partially occurred. For these reasons, we choose to identify suspicious COVID-19 passengers according to the breath score computed through face features. The BS is the most reliable indication of a suspicious COVID-19 passenger.

The respiratory system ensures airflow. The breathing phase is delimited into two steps. The first one enters oxygen to the cell through inhalation. The exhalation step goes out of the carbon dioxide from the intracellular.

The estimation of the Respiratory Rate (RR) is the purpose of many devices. This rate is an important key to evaluate the health condition of a patient and it is a primary factor to predict persons infected by the COVID-19 virus [8].

The COVID-19 is characterized by three essential symptoms: fever, cough, and shortness of breath [7,9]. The need for continuous surveillance of shortness breath is increasingly requested in the healthcare system [10]. Many attempts are proposed in the literature to provide a portable or wearable devices to estimate/compute the RR.

Pulse oximeters devices characterized by their noninvasiveness, availability, and cost-effectiveness are widely used to evaluate the respiratory status of patients [11]. The shortcoming of these devices is the late detection of respiratory depression [12]. Recently, some devices are developed according to advanced signal processing from photoplethysmography [13]. Another accurate non-invasive method is performed to monitor the respiration based on changes measuring variations of thoracic electrical impedance. These attempts not only provide the RR but also compute the ventilation factor [14]. Unfortunately, methods based on impedance suffer from the accuracy of the RR when the rate is small. Therefore, this method is not suitable in the case of shortness of breath [11].

Devices using piezoelectric sensors are developed to estimate the RR through the motion of the chest and abdomen [15].

Acoustic transducers sensors tied to the throat are used to compute the RR. These devices achieve high accuracy [7, 9]. The RR accuracy decreases when noise is added to the acoustic signal such as coughs, snores, cries or sneezes [16].

Revolutionary devices based on contactless methods are proposed. Optical cameras and radars are used to supervise the activities of the respiratory system. The authors in [17, 18] use a thermal camera to estimate the RR according to variations of colors of the skin of patients. RR can be determined via camera either radar that supervises the movement of the chest through tracking pixels' coordinates [19]. The motion of bodies constitutes a great challenge for the accuracy of the RR.

A recent attempt described in [20] presents a non-invasive method to measure the RR. The rate is computed using the thermal transfer between the breathing airflow under the nose and the mouth.

This paper tries to identify an infected passenger with COVID-19 based on the Breath Score (BS) as an indication of the shortness of breath. The proposed SRC system is implemented on Raspberry PI4 board.

Most airports used a thermal camera to read the passenger's temperature to identify suspicious ones. Unfortunately, some passengers who did not want to be confined take paracetamol to avoid the fever. Coughs have partially occurred. For these reasons, we choose to identify suspicious COVID-19 passengers according to the breath score computed through face features. The BS is the most reliable indication of a suspicious COVID-19 passenger.

Section 2 describes the proposed method to estimate the BS based on the video streaming of the face. Section 3 highlights the achieved results performed by the proposed system. Finally, Sect. 4 concludes the paper and proposes some perspectives.

\section{Method}

Provide an accurate tool to predict suspicious COVID-19 persons helps managers at airports specially to separate between healthy passengers and infected ones.

The proposed method aims to recognize the shortness of breath as an indication of a suspect COVID-19 person. We attempt to find the breath score according to a face basedvideo. The method is composed of two phases as mentioned in Fig. 1.

The first phase estimates the heart rate by following these steps: (a) find the forehead as the best Region Of Interest (ROI), (b) apply the Fast Fourier Transformer (FFT) to move from spatial description to temporal one, (c) apply in cascade the Band Pass filter, Eulerian transformer, and Lagrangian transformer. The heart rate is the result of these steps. This phase is described in-depth in our previous work [21].

The second phase provides the Respiration Rate (RR) using the heart rate provided by the first phase [22]. The RR is computed using two parameters as shown in Eq. 1: the HR and the Pulse-Respiration Quotient (PQR).

$R R(t)=H R(t) \times P R Q(t)$

As described in [22], PRQ depends on different factors: sex, age, behavior, psychology, body posture, environmental influences, and health status. The PRQ normalization is 


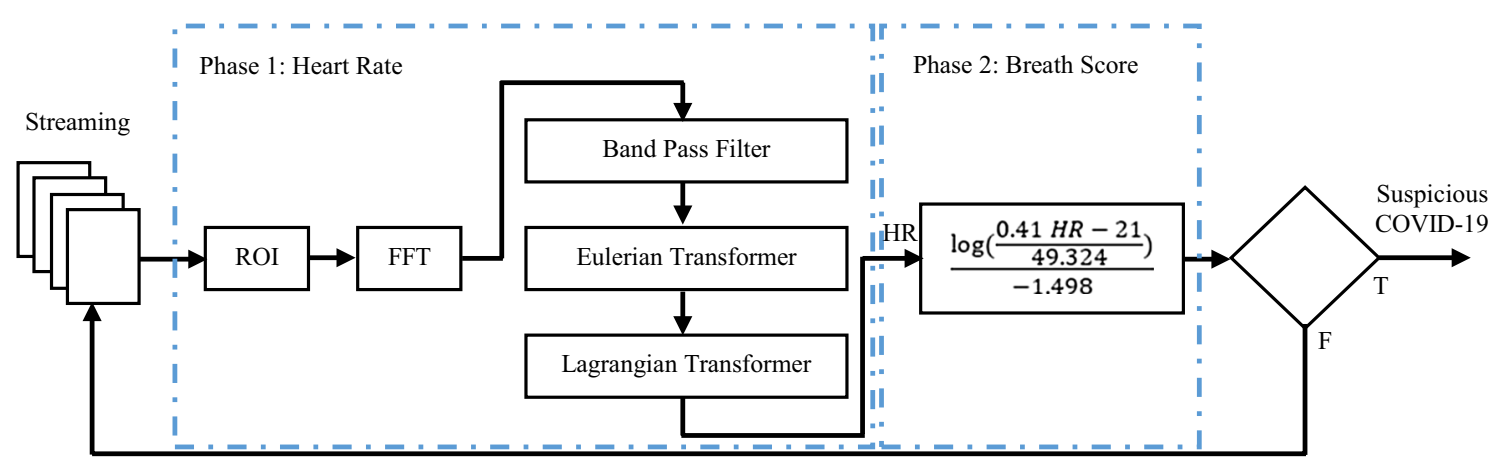

Fig. 1 Description of the SRC System. a Relationship between PQR and HR. b Relationship between PQR and RR

defined as the optimal ratio concerning the functioning of the cardiovascular. Figure 2 defines the PRQ normalized based on the HR (Fig. 2.a) and the RR (Fig. 2.b).

The relationship between the PRQ and HR can be defined by a linear equation. The second curve (b) can be defined by an exponential equation, see Eq. 2. Equation 3 is computed through the Eq. 2 to provide an expression of RR using only the HR.

$\left\{\begin{array}{c}P R Q=25 H R-21 \\ P R Q=49.324 e^{-1.498 R R}\end{array}\right.$

$R R=\frac{\log \left(\frac{0.41 H R-21}{49.324}\right)}{-1.498}$

Figure 3 presents the curve of the RR based on HR. The breath score is computed for $1 \mathrm{~min}$. The curve proves that when the heart rate is increasing the BS is decreased. A suspicious COVID-19 person is identified when the BR is inferior to 10 .

\section{Results}

The SRC system is implemented as a CAD tool to estimate the BS using the face features extracted from video streaming. Figure 4 shows the implementation results of the SRC system developed using Python language associated with the OpenCV library for video processing.

To evaluate the accuracy of the achieved results of the CAD, 10 participants use it. Each one provides ten measurements. These participants are chosen with the following criterion: equitable sex five, adults ([30,60] range), healthy person, and multi-status.

The computed results through the SRC system are compared with Breath Counter Application found in the Play Store developed by Vadion company [23]. The Mean Absolute Error (MAE) and the Mean Squared Error (MSA) are computed for evaluation.

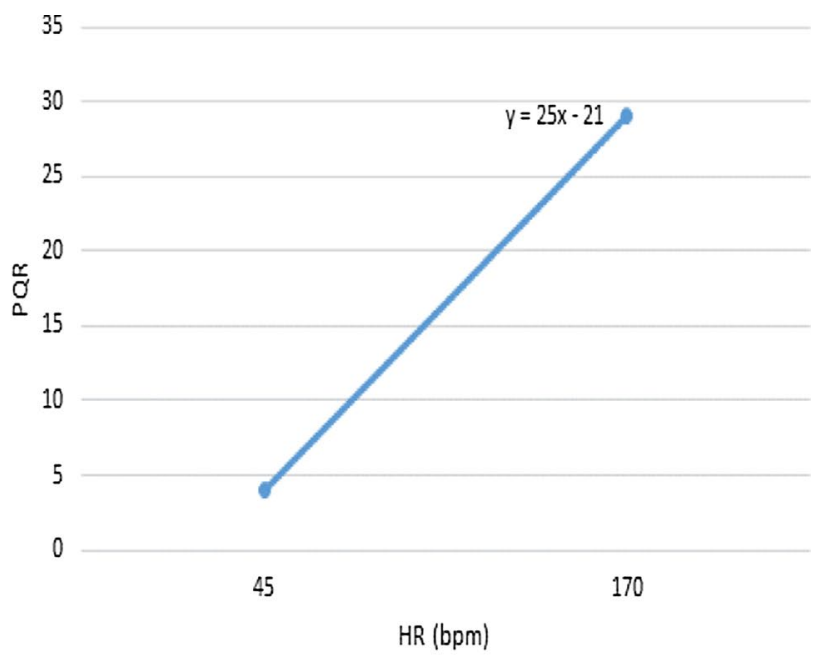

(a) Relationship between PQR and HR

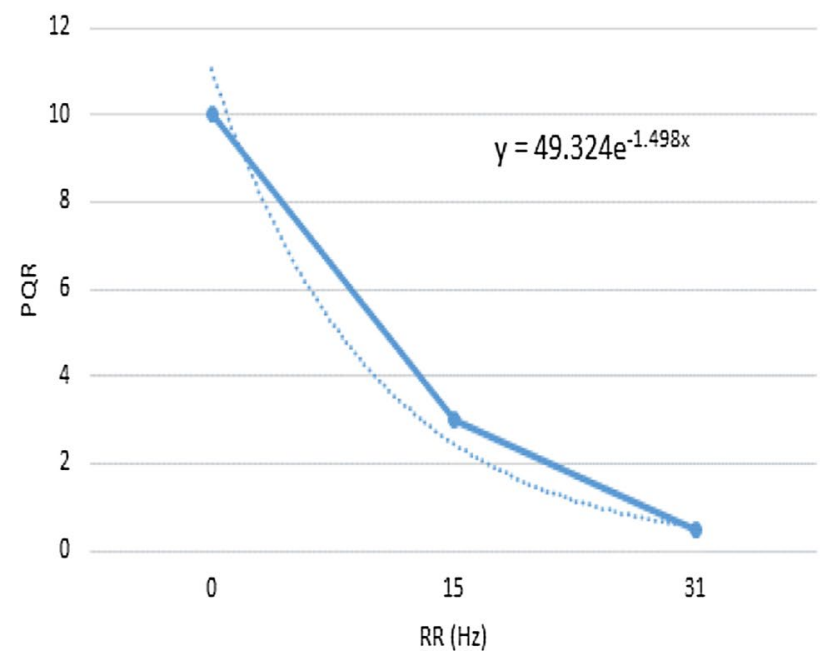

(b) Relationship between PQR and RR

Fig. 2 PQR curves according to the HR and the RR. a The RR curve based on HR. b The BR curve based on HR 


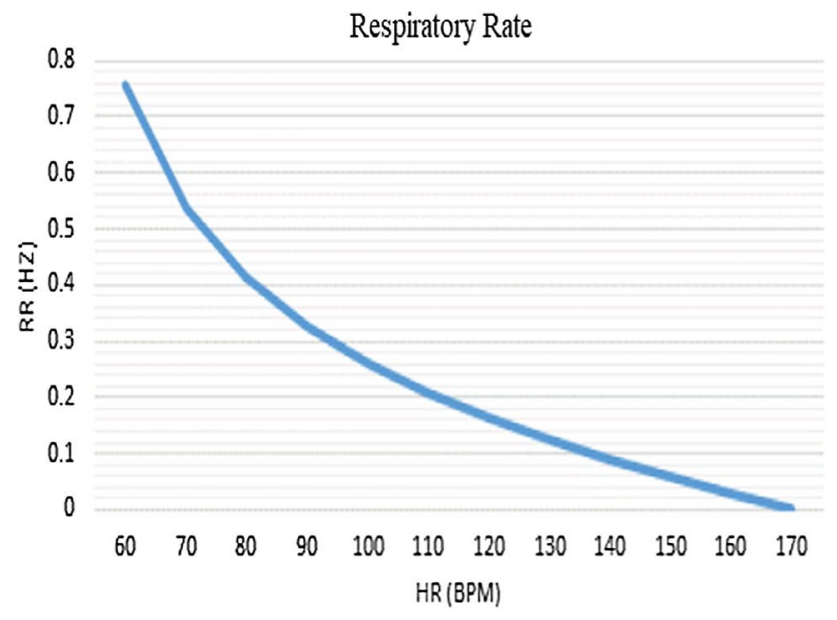

(a) The RR curve based on HR

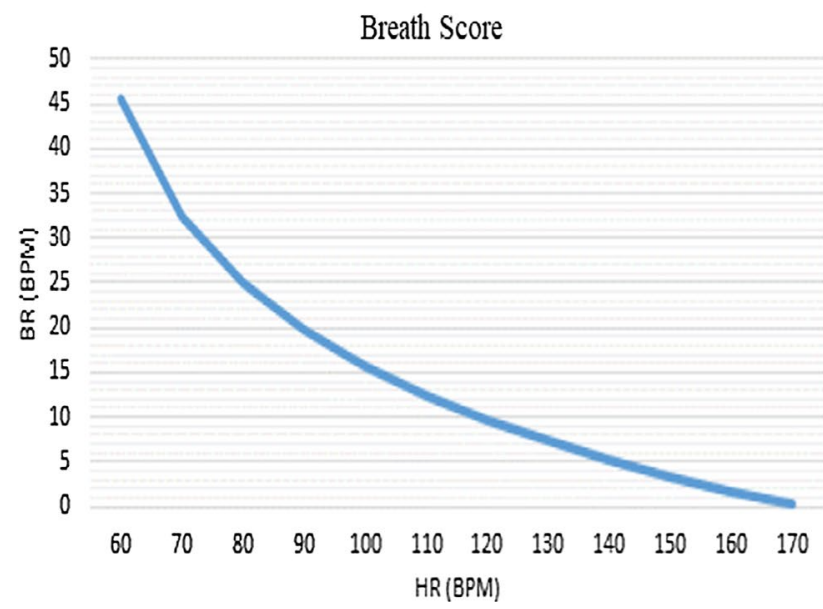

(b) The BR curve based on HR

Fig. 3 Relationship between the RR, the BR, and the HR

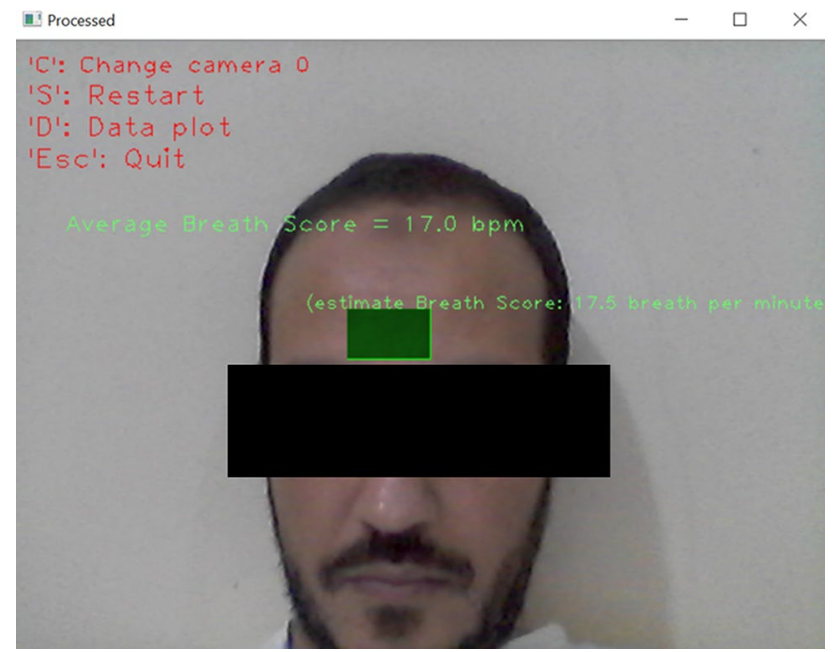

Fig. 4 The SRC system
The Mean Absolute Error (MAE) [24] computes the average of all absolute errors which is defined by Eq. 4 .

The Mean Squared Error (MSE) [24] computes the average of all errors related to the distance between the regression line and the value, see Eq. 5. When the MSE is smaller, the accuracy is higher.

$M A E=\frac{1}{n} \sum_{i=1}^{n}\left|X_{i}-X\right|$

$M S E=\frac{1}{n} \sum_{i=1}^{n}\left(X_{i}-X\right)^{2}$

where $\mathrm{x}$ is the true value provided by the Breath Counter App and $x i$ is the measured value.

Figure 5 describes all attempts done based on the SRC measures and Breath Counter App measures. Curves present a higher rate of overlap. According to these results, Table 1 shows the MAE and the MSE metrics to evaluate the accuracy of the proposed SRC system. Most MAE is inferior to 1 and the MSE belongs to [0.5,2] range. The computed averages of the MAE and the MSE prove that the error is about 1 breath per minute. This is an accurate result which managers and deciders could rely on to identify respiratory problems and especially the case of COVID-19 disease.

Figure 6 shows curves associated with the MAE and the MSE metrics related to each participant. Some cases present an increased error (P1, P5, and P10). This is could be justified by the psychological factors which are not considered in the present paper.

In light of this discussion, we announce that the achieved results are with high accuracy. The SRC system estimates

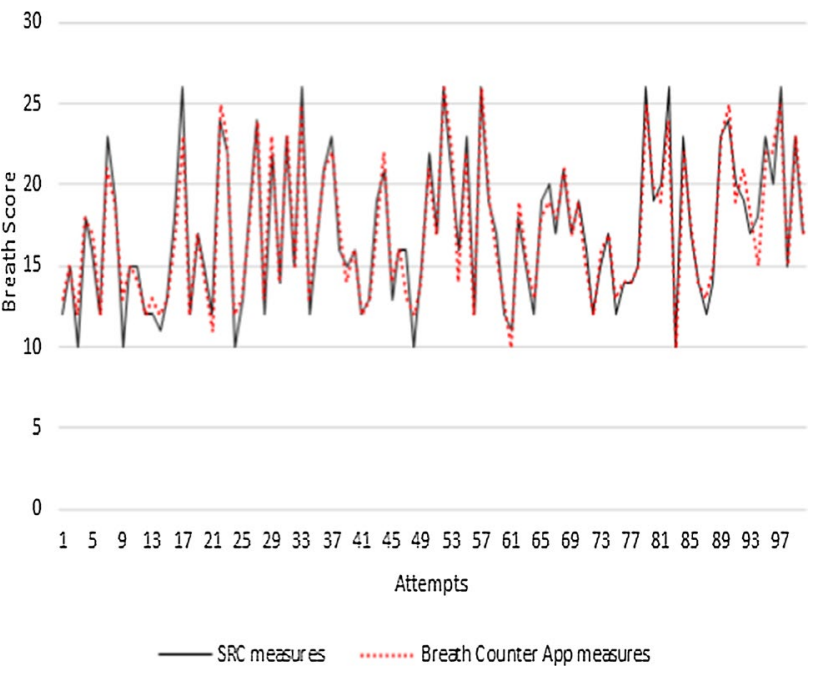

Fig. 5 Breath scores related to all attempts based on SRC measures and Breath Counter App measures 
Table 1 The MAE and MSE of the SRC system

\begin{tabular}{lll}
\hline & MAE & MSE \\
\hline P1 & 0.9 & 1.9 \\
P2 & 0.8 & 1.4 \\
P3 & 0.7 & 0.9 \\
P4 & 0.5 & 0.5 \\
P5 & 1 & 1.8 \\
P6 & 0.6 & 0.8 \\
P7 & 0.6 & 0.6 \\
P8 & 0.5 & 0.5 \\
P9 & 0.8 & 0.1 \\
P10 & 1.1 & 2.1 \\
Average & 0.75 & 1.06 \\
\hline
\end{tabular}

$=$ MAE $=$ MSE

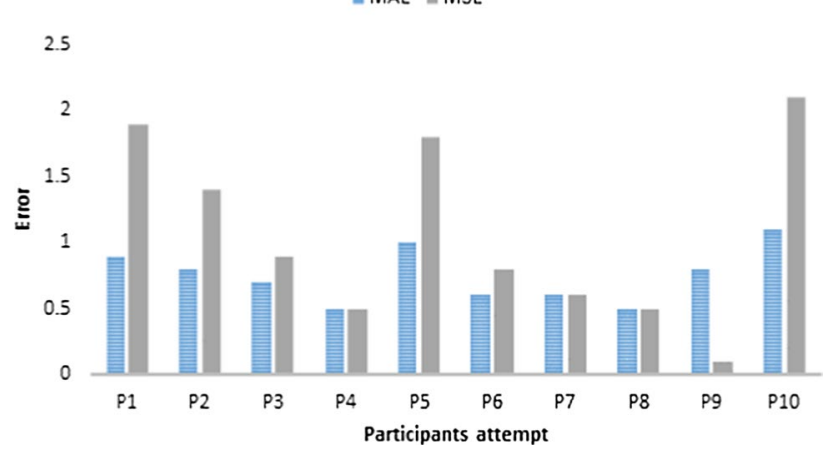

Fig. 6 MAE and MSE errors related to attempts

Table 2 Hardware performances of the SRC system

\begin{tabular}{ll}
\hline & Average \\
\hline Memory usage & $56 \mathrm{MB}$ \\
CPU usage & $92 \%$ \\
CPU load & 1.7 processes / s \\
CPU temperature & $88^{\circ} \mathrm{C}$ \\
GPU temperature & $92{ }^{\circ} \mathrm{C}$ \\
\hline
\end{tabular}

the breath score accurately. Therefore, this CAD tool could help airport managers to recognize suspicious COVID-19 persons and decrease the rate of infections.

The SRC system is implemented on the Raspberry PI4 board to discuss the reliability. We focus on this section on hardware performances which are: (1) the memory usage, (2) the CPU usage, (3) the CPU load, (4) the CPU temperature, and (5) the GPU temperature. Table 2 shows the hardware performances.

The SRC requests a memory size of around $56 \mathrm{MB}$ which is provided by the board. The CPU usage is $92 \%$. A monoprocessor architecture is sufficient in this case. The CPU load corresponds to the number of processes running at the same time per second. There is no need for a bigger buffer because of the CPU load in inferior to 2 processes/s. The temperature of components is a critical hardware factor to avoid the fall-down of the system. The CPU temperature is $88{ }^{\circ} \mathrm{C}$ which is acceptable, but the GPU temperature $\left(92{ }^{\circ} \mathrm{C}\right)$ needs a cooling mechanism to be mounted.

\section{Conclusion}

Since the epidemic COVID-19 virus that swept the whole world, many studies are involved in different disciplines. All studies prove that this virus damages the respiratory system. Therefore, during this paper, we attempt to estimate the breath score via face based-video related to passengers. Based on the BS a passenger could be suspicious COVID19. To achieve our objective, we computed at first the heart rate. Then according to the normalized PQR, the SRC system computes the BS. Achieved results show an accurate estimation in which the error is about 1 breath per minute. The hardware implementation on a raspberry pi 4 board proves the high reliability of the proposed SRC system.

This work would be enhanced by supporting all factors related to PQR and implement the SRC system into an embedded system.

Acknowledgments The authors would like to thank Deanship of Scientific Research at Majmaah University for funding this project under the number R-2021-2.

Data Availability The authors certify that the data that support the findings of this study are available on request from the corresponding author. The data are not publicly available due to their containing information that could compromise the privacy of research participants.

Code Availability The code is not publicly available. It can be requested from the corresponding author.

\section{Compliance with Ethical Standards}

Conflict of interests The authors declare that there is no conflict of interest regarding the publication of this paper.

Human and Animal Participants All procedures performed in studies involving human participants were in accordance with the ethical standards of the institutional and/or national research committee and with the 1964 Helsinki declaration and its later amendments or comparable ethical standards.

\section{References}

1. Bai Y, Yao L, Wei T, Tian F, Jin D-Y, Chen L, Wang M (2020) Presumed asymptomatic carrier transmission of COVID-19. Jama 323(1406): 1407

2. Worldometer. "Corona virus cases," https://www.worldomete rs.info/coronavirus/. 
3. Li Q, Guan X, Wu P, Wang X, Zhou L, Tong Y, Ren R, Leung KS, Lau EH, Wong JY (2020) Early transmission dynamics in Wuhan, China, of novel coronavirus-infected pneumonia. New Engl J Med 382(1199): 1207

4. Koo JR, Cook AR, Park M, Sun Y, Sun H, Lim JT, Tam C, Dickens BL (2020) Interventions to mitigate early spread of SARSCoV-2 in Singapore: a modelling study. Lancet Infectious Dis 20(678):688

5. Chan JF-W, Yuan S, Kok K-H, To KK-W, Chu H, Yang J, Xing F, Liu J, Yip CC-Y, Poon RW-S (2020) A familial cluster of pneumonia associated with the 2019 novel coronavirus indicating personto-person transmission: a study of a family cluster. The Lancet 395(10223):514-523

6. W. H. Organization, "Coronavirus disease 2019 ( COVID-19): situation report, 51," 2020.

7. Wang D, Hu B, Hu C, Zhu F, Liu X, Zhang J, Wang B, Xiang H, Cheng Z, Xiong Y (2020) Clinical characteristics of 138 hospitalized patients with 2019 novel coronavirus-infected pneumonia in Wuhan, China. Jama 323(1061):1069

8. Knaus WA, Zimmerman JE, Wagner DP, Draper EA, Lawrence DE (1981) APACHE-acute physiology and chronic health evaluation: a physiologically based classification system. Crit Care Med 9(8):591-597

9. Guechi Y, Pichot A, Frasca D, Rayeh-Pelardy F, Lardeur J-Y, Mimoz O (2015) Assessment of noninvasive acoustic respiration rate monitoring in patients admitted to an emergency department for drug or alcoholic poisoning. J Clin Monit Comput 29(6):721-726

10. Hogan J (2006) Why don't nurses monitor the respiratory rates of patients? Br J Nurs 15(9):489-492

11. Folke M, Cernerud L, Ekström M, Hök B (2003) Critical review of non-invasive respiratory monitoring in medical care. Med Biol Eng Comput 41(4):377-383

12. Zhang X, Kassem MAM, Zhou Y, Shabsigh M, Wang Q, Xu X (2017) A brief review of non-invasive monitoring of respiratory condition for extubated patients with or at risk for obstructive sleep apnea after surgery. Front Med 4:26

13. Addison PS, Watson JN, Mestek ML, Ochs JP, Uribe AA, Bergese SD (2015) Pulse oximetry-derived respiratory rate in general care floor patients. J Clin Monit Comput 29(1):113-120

14. Cavalcante AN, Martin YN, Sprung J, Imsirovic J, Weingarten TN (2018) Low minute ventilation episodes during anesthesia recovery following intraperitoneal surgery as detected by a non-invasive respiratory volume monitor. J Clin Monit Comput 32(5):929-935

15. Lee PJ (2016) Clinical evaluation of a novel respiratory rate monitor. J Clin Monit Comput 30(2):175-183

16. Güder F, Ainla A, Redston J, Mosadegh B, Glavan A, Martin T, Whitesides GM (2016) Paper-based electrical respiration sensor. Angew Chem Int Ed 55(19):5727-5732

17. Hochhausen N, Barbosa Pereira C, Leonhardt S, Rossaint R, Czaplik M (2018) Estimating respiratory rate in post-anesthesia care unit patients using infrared thermography: an observational study. Sensors 18(5):1618-1619

18. van Gastel M, Stuijk S, de Haan G (2016) Robust respiration detection from remote photoplethysmography. Biomed Opt Exp 7(12):4941-4957

19. Li MH, Yadollahi A, Taati B (2016) Noncontact vision-based cardiopulmonary monitoring in different sleeping positions. IEEE $\mathrm{J}$ Biomed Health Info 21(5):1367-1375

20. Hurtado DE, Abusleme A, Chávez JA (2019) Non-invasive continuous respiratory monitoring using temperature-based sensors. J Clin Monit Comput 34:1-9
21. Ayed MB, Elkosantini S, Alshaya SA, Abid M (2019) Suspicious behavior recognition based on face features. IEEE Access 7:149952-149958

22. Scholkmann F, Wolf U (2019) The pulse-respiration quotient: a powerful but untapped parameter for modern studies about human physiology and pathophysiology. Front Physiol 10:371

23. Vadion. "Breath Counter," https://vadion.com/.

24. Jachner S, Van den Boogaart G, Petzoldt T (2007) Statistical methods for the qualitative assessment of dynamic models with time delay (R Package qualV). J Stat Softw 22(8):1-30

Publisher's Note Springer Nature remains neutral with regard to jurisdictional claims in published maps and institutional affiliations.

Dr. Mossaad Ben Ayed He received recently the HDR degree in 2019 in Embedded systems. He obtained the Phd diploma in embedded system in the field of simulation in 2013. In 2007, he achieved the electrical engineering degree. From 2007, he was an active researcher at the Laboratory of Computer Embedded System (CES), National Engineering School of Sfax, Tunisia (http://ceslab.org/fr/perso.php?id=81). He is currently an assistant professor in college of science and humanities at alGhat, Majmaah University, Riyadh, Kingdom of Saudi Arabia. His field of research includes Embedded system, Simulation, Computer-Aided design, Emulation, Modelling, Co-design, and Pattern Recognition.

Dr. Ayman Massaoudi (IEEE Member, IEEE Communications Society Member) is an Assistant Professor at Jouf University, Kingdom of Saudi Arabia. He received his $\mathrm{PhD}$ in Information and Communication Technologies (ICT) from the Higher School of Communication of Tunis (SupCom), University of Carthage, Tunisia in 2016. Dr. Massaoudi also received the Master of Sciences degree and the engineering degree in Telecommunications from the SupCom, University of Carthage, Tunisia in 2009 and 2008 respectively. He is a member of MEDIATRON research Lab, SupCom, Tunisia. Dr. Massaoudi current research interests include communication systems, embedded Systems, diversity techniques, MIMO techniques, cognitive radio systems, 5G networks and Internet of Think (IoT). He has published several papers on the above subjects published in IEEE conferences including VTC, PIMRC, EUSIPCO, ComNet. He has won several Best Paper Awards in prestigious conferences.

Dr. Shaya Alshaya Coming from the invaluable educational background in both under and postgraduate degrees from Saudi Arabia, USA, and Italy, Dr. Alshaya has put his knowledge into practicality as an international entrepreneur, his endeavors include education and research, ICT sector. He is a member of the National Association of Industrial Technology, the Institute of Electrical Engineers and the Sloan-C Online Association amongst others. Dr. Alshaya has a wealthy experience as Head of IT in Majmaah University, Saudi Arabia. His experience as a researcher, consultant, project manager and information technologist has allowed him to bring his extensive experience of technology planning, training and implementation to all of the projects he endeavors. The numerous national and international companies that utilize his expertise as a consultant evidence Dr. Alshaya's success. Dr. Alshaya's wealthy experience, vast knowledge is integral to his vision and future projects. 